\title{
Higher-order soft and Coulomb corrections to squark and gluino production at the LHC
}

\author{
Martin Beneke \\ Physik Department T31, James-Franck-Straße, Technische Universität München, \\ D-85748 Garching, Germany
}

\section{Pietro Falgari}

Institute for Theoretical Physics and Spinoza Institute, Utrecht University, 3508 TD Utrecht, The Netherlands

\section{Jan Piclum ${ }^{* \dagger}$}

Physik Department T31, James-Franck-Straße, Technische Universität München, D-85748 Garching, Germany

and

Institut für Theoretische Teilchenphysik und Kosmologie, RWTH Aachen University, D-52056 Aachen, Germany

E-mail: jan.piclumetum.de

\section{Christian Schwinn}

Albert-Ludwigs Universität Freiburg, Physikalisches Institut, D-79104 Freiburg, Germany

\section{Christopher Wever}

Institute of Nuclear Physics, NCSR "Demokritos", GR 15310 Athens, Greece

\begin{abstract}
We present predictions for the total cross sections for pair production of squarks and gluinos at the LHC based on a combined resummation of soft and Coulomb gluon effects at next-to-next-toleading logarithmic (NNLL) order. The NNLL corrections can be up to $25 \%$ relative to previous NLL results and reduce the theoretical uncertainties to the $10 \%$ level.
\end{abstract}

Loops and Legs in Quantum Field Theory - LL 2014,

27 April - 2 May 2014

Weimar, Germany

\footnotetext{
${ }^{*}$ Speaker.

${ }^{\dagger}$ Supported by the DFG Sonderforschungsbereich/Transregio 9 "Computergestützte Theoretische Teilchenphysik"

‡Partially funded by Research Funding Program ARISTEIA, HOCTools (co-financed by the European Union (European Social Fund ESF) and Greek national funds through the Operational Program "Education and Lifelong Learning" of the National Strategic Reference Framework (NSRF)) and by the research programme Mozaiek (NWO)
} 


\section{Introduction}

Supersymmetry (SUSY) and its realization in the $R$-parity conserving Minimally Supersymmetric Standard Model is a well-studied and motivated extension of the Standard Model (SM). It could provide a solution to shortcomings of the SM such as the absence of a dark matter candidate and it might stabilize the electroweak scale against quantum corrections. The search for SUSY at the $\mathrm{TeV}$ scale is therefore a central part of the physics program of the Large Hadron Collider (LHC). At hadron colliders, the production of squarks and gluinos, the superpartners of quarks and gluons, is expected to be the dominant signature. Current LHC limits exclude gluino masses up to $m_{\tilde{g}}=1.3 \mathrm{TeV}$ and superpartners of the quarks of the first two generations below $m_{\tilde{q}} \lesssim 850 \mathrm{GeV}$. Equal squark and gluino masses can be excluded up to $m_{\tilde{g}} \sim 1.7 \mathrm{TeV}$ [1]. However, these bounds depend on assumptions e.g. on the mass of the lightest supersymmetric particle and can be evaded for instance by compressed mass spectra. The search for SUSY will therefore remain a focus of the 13-14 TeV run of the LHC that has the potential to discover or exclude squarks and gluinos in the $3 \mathrm{TeV}$ range. Turning exclusion limits on production cross sections into bounds on superparticle masses requires precise predictions for these cross sections. In this contribution we report on the status of predictions for squark and gluino production at the LHC and present results of a combined NNLL resummation of soft-gluon and Coulomb corrections [约].

\section{Squark and gluino production at the LHC}

At hadron colliders, light-flavour squarks and gluinos, denoted jointly by $\tilde{s}, \tilde{s}^{\prime} \in\{\tilde{q}, \overline{\tilde{q}}, \tilde{g}\}$, can be pair-produced through partonic production processes of the form $p p^{\prime} \rightarrow \tilde{s} \tilde{s}^{\prime} X$ from the incoming partons $p, p^{\prime} \in\{q, \bar{q}, g\}$. The relevant production channels at leading order (LO) are

$$
g g, q \bar{q} \rightarrow \tilde{q} \overline{\tilde{q}}, \quad q q \rightarrow \tilde{q} \tilde{q}, \quad g q \rightarrow \tilde{g} \tilde{q}, \quad g g, q \bar{q} \rightarrow \tilde{g} \tilde{g},
$$

and the corresponding charge-conjugated channels for squarks. Flavour indices of quarks and squarks have been suppressed. We use a common mass $m_{\tilde{q}}$ for the light-flavour squarks and do not consider the production of stop pairs which has been discussed in [3]. In the upper plots in Figure 1 the relative contribution of the processes (2.1) to the inclusive squark and gluino production cross section $\sigma_{\mathrm{SUSY}}=\sigma_{\tilde{q} \tilde{q}+\tilde{q} \tilde{q}+\tilde{g} \tilde{q}+\tilde{g} \tilde{g}}$ is shown for the LHC with $\sqrt{s}=8 \mathrm{TeV}$ centre-of-mass energy. The left-hand plot displays the relative contributions of the processes (2.1) as a function of a common squark and gluino mass, while in the right-hand plot the relative contributions are shown as a function of the squark-gluino mass ratio. The results for the $K$-factor $K_{\mathrm{NLO}}=\sigma_{\mathrm{NLO}} / \sigma_{\mathrm{LO}}$ for the next-toleading order (NLO) SUSY-QCD corrections [4] obtained with the program PROSP INO [F] in the lower plots in Figure 1 show that the NLO corrections can be of the order of $100 \%$ of the tree-level cross section.

The large NLO K-factors can be attributed to the enhancement of radiative corrections in the threshold limit $\beta \equiv \sqrt{1-4 M^{2} / \hat{s}} \rightarrow 0$, with the average sparticle mass $M=\frac{1}{2}\left(m_{\tilde{s}}+m_{\tilde{S}^{\prime}}\right)$ and the partonic centre-of-mass energy $\hat{s}$. In this limit the partonic cross section is dominated by soft-gluon emission off the coloured initial- and final-state particles and by Coulomb interactions of the two non-relativistic heavy sparticles in the final state, which give rise to singular terms of the form $\alpha_{s} \ln ^{2,1} \beta$ and $\alpha_{s} / \beta$, respectively. The radiative corrections in the threshold limit can be written in 

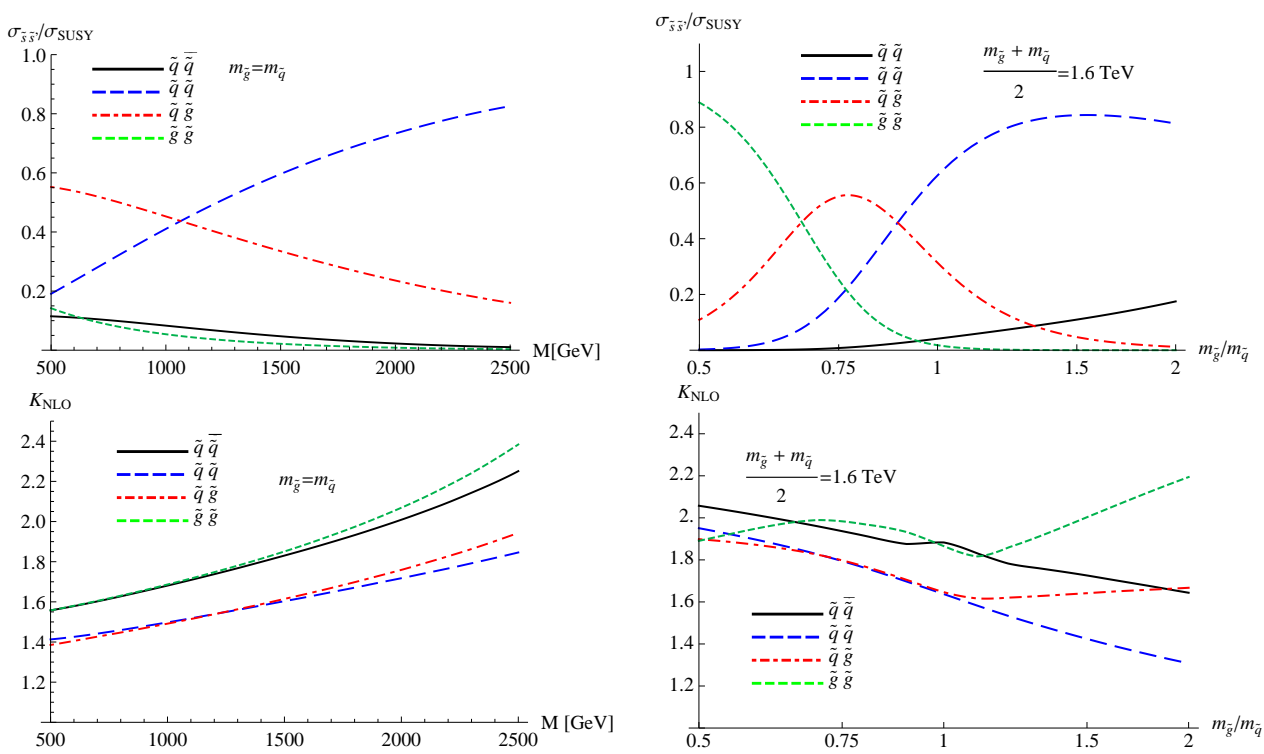

Figure 1: Top: Relative contribution of the different squark and gluino production processes to the total Born production rate of coloured sparticles, $\sigma_{\mathrm{SUSY}}$, for the LHC with $\sqrt{s}=8 \mathrm{TeV}$. Below: NLO $K$-factor for squark and gluino production processes at the LHC. The left plots show the mass dependence for $m_{\tilde{q}}=m_{\tilde{g}}=$ $M$ while the right plot shows the dependence on the ratio $m_{\tilde{g}} / m_{\tilde{q}}$ for a fixed average mass $\left(m_{\tilde{q}}+m_{\tilde{g}}\right) / 2=$ 1.6 TeV. In the $K$-factors the MSTW2008NLO PDFs [6] have been used for the LO and NLO cross sections.

a simple and process-independent form using a colour decomposition of the total partonic cross section,

$$
\hat{\sigma}_{p p^{\prime}}\left(\hat{s}, \mu_{f}\right)=\sum_{R_{\alpha}} \hat{\sigma}_{p p^{\prime}}^{(0), R_{\alpha}}\left(\hat{s}, \mu_{f}\right)\left\{1+\frac{\alpha_{s}}{4 \pi} f_{p p^{\prime}}^{(1), R_{\alpha}}\left(\hat{s}, \mu_{f}\right)+\ldots\right\},
$$

where $\mu_{f}$ is the factorization scale, $R_{\alpha}$ are the irreducible representations in the decomposition $R \otimes R^{\prime}=\sum R_{\alpha}$ of the product of the $S U(3)$ representations $R$ and $R^{\prime}$ of the two final-state sparticles,

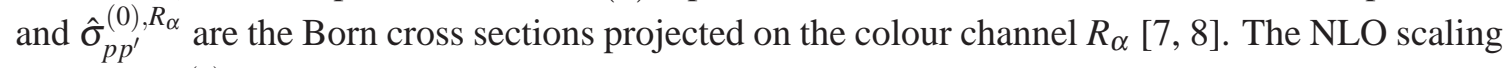
functions $f_{p p^{\prime}}^{(1), R_{\alpha}}$ assume a simple form in the threshold limit [9]:

$$
\begin{aligned}
f_{p p^{\prime}}^{(1), R_{\alpha}}\left(\hat{s}, \mu_{f}\right)= & -\frac{2 \pi^{2} D_{R_{\alpha}}}{\beta} \sqrt{\frac{2 m_{r}}{M}}+4\left(C_{r}+C_{r^{\prime}}\right)\left[\ln ^{2}\left(\frac{8 M \beta^{2}}{\mu_{f}}\right)+8-\frac{11 \pi^{2}}{24}\right] \\
& -4\left[C_{R_{\alpha}}+4\left(C_{r}+C_{r^{\prime}}\right)\right] \ln \left(\frac{8 M \beta^{2}}{\mu_{f}}\right)+12 C_{R_{\alpha}}+h_{p p^{\prime}}^{(1), R_{\alpha}}+\mathscr{O}(\beta) .
\end{aligned}
$$

Here $m_{r}=m_{\tilde{s}} m_{\tilde{s}^{\prime}} /\left(m_{\tilde{s}}+m_{\tilde{s}^{\prime}}\right)$ is the reduced mass, $r$ and $r^{\prime}$ are the colour representations of the initial partons $p$ and $p^{\prime}$, and $C_{R}$ is the quadratic Casimir invariant for a representation $R$. The coefficients of the Coulomb potential for sparticles in the representations $R$ and $R^{\prime}$ in the colour channel $R_{\alpha}$ read $D_{R_{\alpha}}=\frac{1}{2}\left(C_{R_{\alpha}}-C_{R}-C_{R^{\prime}}\right)$ where negative (positive) values correspond to an attractive (repulsive) potential. The process-dependent coefficients $h_{p p^{\prime}}^{(1), R_{\alpha}}$ have been recently computed for all squark and gluino production processes [10-13]. The singular threshold corrections, i.e. all terms in (2.3) apart from constants, usually dominate the total NLO cross section and differ from the exact NLO result by typically only $10-30 \%$ [ [ 3 . This motivates the computation of the higher-order thresholdenhanced terms, as discussed in the remainder of this contribution. 


\section{Joint soft and Coulomb resummation}

Near the partonic production threshold $\beta \rightarrow 0$ the conventional perturbative expansion in $\alpha_{s}$ breaks down and the perturbative series has to be rearranged by treating both threshold logarithms $\alpha_{s} \ln \beta$ and Coulomb corrections $\alpha_{s} / \beta$ as quantities of order one. The accuracy of the rearranged perturbative series can be defined by representing the resummed cross section schematically as

$$
\hat{\sigma}_{p p^{\prime}}=\hat{\sigma}_{p p^{\prime}}^{(0)} \sum_{k=0}^{\infty}\left(\frac{\alpha_{s}}{\beta}\right)^{k} \exp [\underbrace{\ln \beta g_{0}\left(\alpha_{s} \ln \beta\right)}_{(\mathrm{LL})}+\underbrace{g_{1}\left(\alpha_{s} \ln \beta\right)}_{(\mathrm{NLL})}+\underbrace{\alpha_{s} g_{2}\left(\alpha_{s} \ln \beta\right)}_{(\mathrm{NNLL})}+\ldots]\left\{1+\alpha_{s} c_{\mathrm{NNLL}}+\ldots\right\} .
$$

Methods for the separate resummation of threshold logarithms [14-16] and Coulomb corrections [17] are well known. Applications to squark and gluino production include NLL resummation of threshold logarithms [1], 8, 18, Coulomb resummation [17, 19, 18, 11, approximate NNLO calculations [20, 12], and NNLL resummation of threshold logarithms [10, 21, 2, 22, 23].

The combined NLL resummation of Coulomb and soft effects has been performed for squarkantisquark production in [18] and all other processes in [3], where it was found that Coulomb corrections and soft-Coulomb interference can be as large as the soft corrections alone. In the following, we discuss the extension of this result to NNLL. Up to this accuracy, partonic cross sections in the limit $\beta \rightarrow 0$ factorize into a hard function $H^{R_{\alpha}}$, a soft function $W^{R_{\alpha}}$, and a Coulomb function $J_{R_{\alpha}}$ [24, 18]:

$$
\hat{\sigma}_{p p^{\prime}}\left(\hat{s}, \mu_{f}\right)=\sum_{R_{\alpha}} H_{p p^{\prime}}^{R_{\alpha}}\left(m_{\tilde{q}}, m_{\tilde{g}}, \mu_{f}\right) \int d \omega J_{R_{\alpha}}\left(M \beta^{2}-\frac{\omega}{2}\right) W^{R_{\alpha}}\left(\omega, \mu_{f}\right) .
$$

The hard function encodes the partonic hard-scattering processes and is related to squared onshell scattering amplitudes at threshold. The potential function sums the exchange of Coulomb gluons associated with corrections of order $\left(\alpha_{s} / \beta\right)^{n}$ while the soft function sums the threshold logarithms. The convolution of the soft- and potential functions accounts for the energy loss of the squark/gluino system due to soft gluons with energy of the order $M \beta^{2}$. Near threshold, softgluon radiation is only sensitive to the total colour state $R_{\alpha}$ of the non-relativistic squark/gluino system, as has been shown to all orders in the strong coupling [24], consistent with explicit oneloop calculations [15, 7, 8]. The formula (3.2) has been derived for particles dominantly produced in an $S$-wave, i.e. with a cross section $\hat{\sigma} \sim \beta$, which is the case for all production channels of lightflavour squarks and gluinos, and for processes with a leading $P$-wave contribution $\hat{\sigma} \sim \beta^{3}$ [ [ 3 ], as for stop-antistop production from a quark-antiquark initial state.

Resummation of threshold logarithms is performed by evolving the soft function from a soft scale $\mu_{s} \sim M \beta^{2}$ to a hard-scattering scale $\mu_{f} \sim M$ using a renormalization-group equation derived in [24] with results from [25] (equivalent results have been obtained independently in the traditional Mellin-space approach [26]). The hard function is evolved from a scale $\mu_{h} \sim 2 M$ to $\mu_{f}$. In the momentum-space formalism [16] the resummed cross section can be written as

$$
\hat{\sigma}_{p p^{\prime}}^{\mathrm{res}}\left(\hat{s}, \mu_{f}\right)=\sum_{R_{\alpha}} H_{p p^{\prime}}^{R_{\alpha}}\left(\mu_{h}\right) U_{R_{\alpha}}\left(\mu_{h}, \mu_{s}, \mu_{f}\right)\left(\frac{2 M}{\mu_{s}}\right)^{-2 \eta} \tilde{s}^{R_{\alpha}}\left(\partial_{\eta}, \mu_{s}\right) \frac{e^{-2 \gamma_{E} \eta}}{\Gamma(2 \eta)} \int_{0}^{\infty} d \omega \frac{J_{R_{\alpha}}\left(M \beta^{2}-\frac{\omega}{2}\right)}{\omega}\left(\frac{\omega}{\mu_{s}}\right)^{2 \eta}
$$


Resummation at NNLL accuracy requires the expansions of the hard function and the Laplacetransformed soft function [24] up to NLO,

$$
\begin{aligned}
& H_{p p^{\prime}}^{R_{\alpha}}\left(\mu_{h}\right)=H_{p p^{\prime}}^{R_{\alpha}(0)}\left(\mu_{h}\right)\left[1+\frac{\alpha_{s}\left(\mu_{h}\right)}{4 \pi} h_{p p^{\prime}}^{R(1)}\left(\mu_{h}\right)+\mathscr{O}\left(\alpha_{s}^{2}\right)\right] \\
& \tilde{s}^{R_{\alpha}}(\rho, \mu)=\int_{0_{-}}^{\infty} d \omega e^{-s \omega} W^{R_{\alpha}}(\omega, \mu)=1+\frac{\alpha_{s}}{4 \pi}\left[\left(C_{r}+C_{r^{\prime}}\right)\left(\rho^{2}+\frac{\pi^{2}}{6}\right)-2 C_{R_{\alpha}}(\rho-2)\right]+\mathscr{O}\left(\alpha_{s}^{2}\right),
\end{aligned}
$$

with $s=1 /\left(e^{\gamma_{E}} \mu e^{\rho / 2}\right)$. The one-loop hard coefficients are the same as in 2.3 . The functions $U_{R_{\alpha}}$ and $\eta$ contain logarithms of the ratios of the various scales, the explicit expressions at NNLL can be found in [16].

For NNLL accuracy, the NLO potential function is required that can be written as [27]

$$
J_{R_{\alpha}}(E)=2 \operatorname{Im}\left[G_{C, R_{\alpha}}^{(0)}(0,0 ; E) \Delta_{\mathrm{nC}}(E)+G_{C, R_{\alpha}}^{(1)}(0,0 ; E)+\ldots\right],
$$

where $G_{C, R_{\alpha}}^{(0)}$ is the solution to the Schrödinger equation with the leading Coulomb potential, resumming all $\left(\alpha_{s} / \beta\right)^{n}$ corrections. The function $G_{C, R_{\alpha}}^{(1)}$ sums $\alpha_{s} \times\left(\alpha_{s} / \beta\right)^{n}$ corrections by solving perturbatively the Schrödinger equation with one insertion of the NLO Coulomb potential,

$$
\delta \tilde{V}(\mathbf{p}, \mathbf{q})=\frac{4 \pi D_{R_{\alpha}} \alpha_{s}(\mu)}{\mathbf{q}^{2}} \frac{\alpha_{s}(\mu)}{4 \pi}\left(a_{1}-\beta_{0} \ln \frac{\mathbf{q}^{2}}{\mu^{2}}\right),
$$

where $\beta_{0}$ is the one-loop beta-function coefficient, and $a_{1}=\frac{31}{9} C_{A}-\frac{20}{9} n_{l} T_{f}$. The factor $\Delta_{\mathrm{nC}}$ arises from non-Coulomb NNLO potential terms [28]. For squark and gluino production, these read [2]

$$
\begin{aligned}
\delta \tilde{V}_{\mathrm{NNLO}}(\mathbf{p}, \mathbf{q})= & \frac{4 \pi D_{R_{\alpha}} \alpha_{s}\left(\mu^{2}\right)}{\mathbf{q}^{2}}\left[\frac{\pi \alpha_{s}\left(\mu^{2}\right)|\mathbf{q}|}{8 m_{r}}\left(\frac{D_{R_{\alpha}}}{2} \frac{2 m_{r}}{M}+C_{A}\right)\right. \\
& +\frac{\mathbf{p}^{2}}{m_{\tilde{s}} m_{\tilde{S}^{\prime}}}-\frac{\mathbf{q}^{2}}{8 m_{\tilde{S}}^{2} m_{\tilde{S}^{\prime}}^{2}}\left(2 m_{\tilde{s}} m_{\tilde{S}^{\prime}}+m_{\tilde{s}}^{2} c_{2}\left(m_{\tilde{S}^{\prime}}\right)+m_{\tilde{S}^{\prime}}^{2} c_{2}\left(m_{\tilde{S}}\right)\right) \\
& \left.+\frac{1}{16 m_{\tilde{s}} m_{\tilde{s}^{\prime}}}\left[\sigma^{i}, \sigma^{j}\right] q^{j} \otimes\left[\sigma^{i}, \sigma^{k}\right] q^{k}+\ldots\right],
\end{aligned}
$$

where terms not contributing to squark and gluino production processes are not shown. For scalars the spin-dependent terms are set to zero. The matching coefficient $c_{2}$ has the tree-level value zero (one) for scalars (fermions). Projecting on the relevant spin states, the non-Coulomb correction in (3.6) is obtained as

$$
\Delta_{\mathrm{nC}}(E)=1+\alpha_{s}^{2}\left(\mu_{C}\right) \ln \beta\left[-2 D_{R_{\alpha}}^{2}\left(1+v_{\text {spin }}\right)+D_{R_{\alpha}} C_{A}\right] \theta(E),
$$

with the Coulomb scale given by $\mu_{C}=\max \left\{2 m_{r}\left|D_{R_{\alpha}}\right| \alpha_{s}\left(\mu_{C}\right), 2 \beta \sqrt{2 m_{r} M}\right\}[3]$. The spin-dependent coefficient for the squark and gluino production processes is given by

$$
\begin{aligned}
& v_{\text {spin }}(\tilde{q} \overline{\tilde{q}})=v_{\text {spin }}(\tilde{q} \tilde{q})=-\frac{2 m_{r}}{4 M}, \quad v_{\text {spin }}(\tilde{q} \tilde{g})=\frac{1}{2}\left(\frac{m_{\tilde{\tilde{g}}}^{2}}{\left(m_{\tilde{q}}+m_{\tilde{g}}\right)^{2}}-1\right), \\
& v_{\text {spin }}\left((\tilde{g} \tilde{g})_{S=0}\right)=0, \quad v_{\text {spin }}\left((\tilde{g} \tilde{g})_{S=1}\right)=-\frac{2}{3} .
\end{aligned}
$$



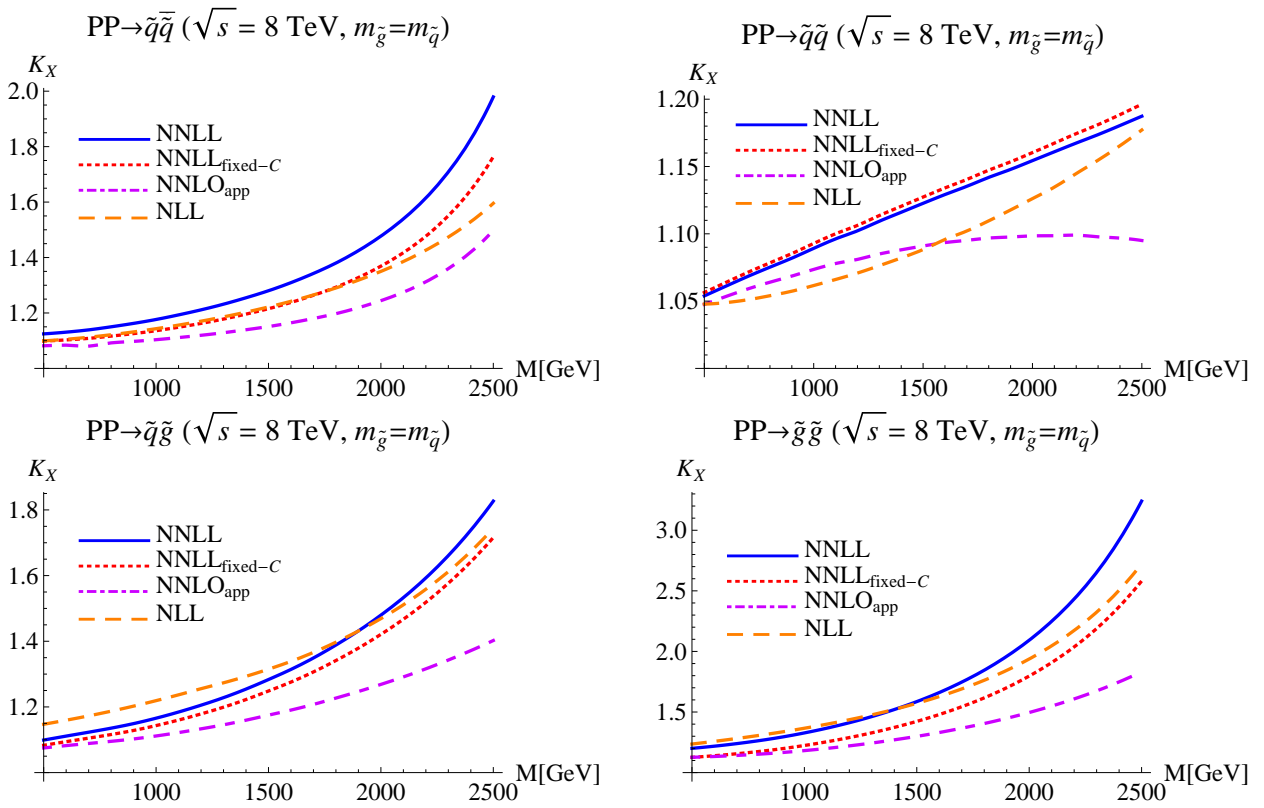

Figure 2: Higher-order corrections relative to the NLO cross section for squark and gluino production at the LHC with $\sqrt{\hat{s}}=8 \mathrm{TeV}$ for full NNLL resummation (solid blue), NNLL with fixed-order Coulomb corrections (dotted red), approximate NNLO (dot-dashed pink), and NLL (dashed orange). The NLL and NLO (NNLO ${ }_{a p p}$ and NNLL) cross sections are computed with the NLO (NNLO) MSTW2008 PDFs.

The gluino pairs are produced with spin $S=0$ for the symmetric colour representations $1,8_{s}, 27$ and with $S=1$ for anti-symmetric colour representations $8_{a}, 10$ (see e.g. [11]). An additional term of the same order has been pointed out recently in the context of top-quark pair production [29]. This term is not yet included in our results, but can be treated in the same way.

In the colour channels with an attractive Coulomb potential, the Coulomb Green function develops bound-state poles below threshold. We include these bound-state contributions and convolute them with the soft corrections as described in [27]. If the finite decay width of squarks and gluinos is taken into account, the bound-state poles are smeared out. This has been investigated at NLL accuracy in [30] with the conclusion that for $\Gamma_{\tilde{s}} / m_{\tilde{s}} \lesssim 5 \%$ the uncertainties due to finite width effects are smaller than the uncertainties of the NLL calculation.

\section{Squark and gluino production at NNLL}

We have implemented the NNLL resummation discussed in Section 3 following the treatment of top-quark pair production in [27, 31]. A public program based on topixs [31] is in preparation. As in the previous NLL resummation [3], the LO hard functions are expressed in terms of the exact colour separated Born cross sections. No resummation is performed for colour channels that are suppressed at threshold. The convolution of the resummed partonic cross section with the PDFs is regularized as discussed in [27]. The NNLL cross section is matched to the sum of the exact NLO cross section [ [ ] from PROSP INO and the approximate NNLO correction [9] where double counting is avoided by subtracting the NNLO-expansion of the resummed cross section. In order to see the impact of Coulomb resummation, we also consider an approximation $\mathrm{NNLL}_{\text {fixed-C }}$ where 

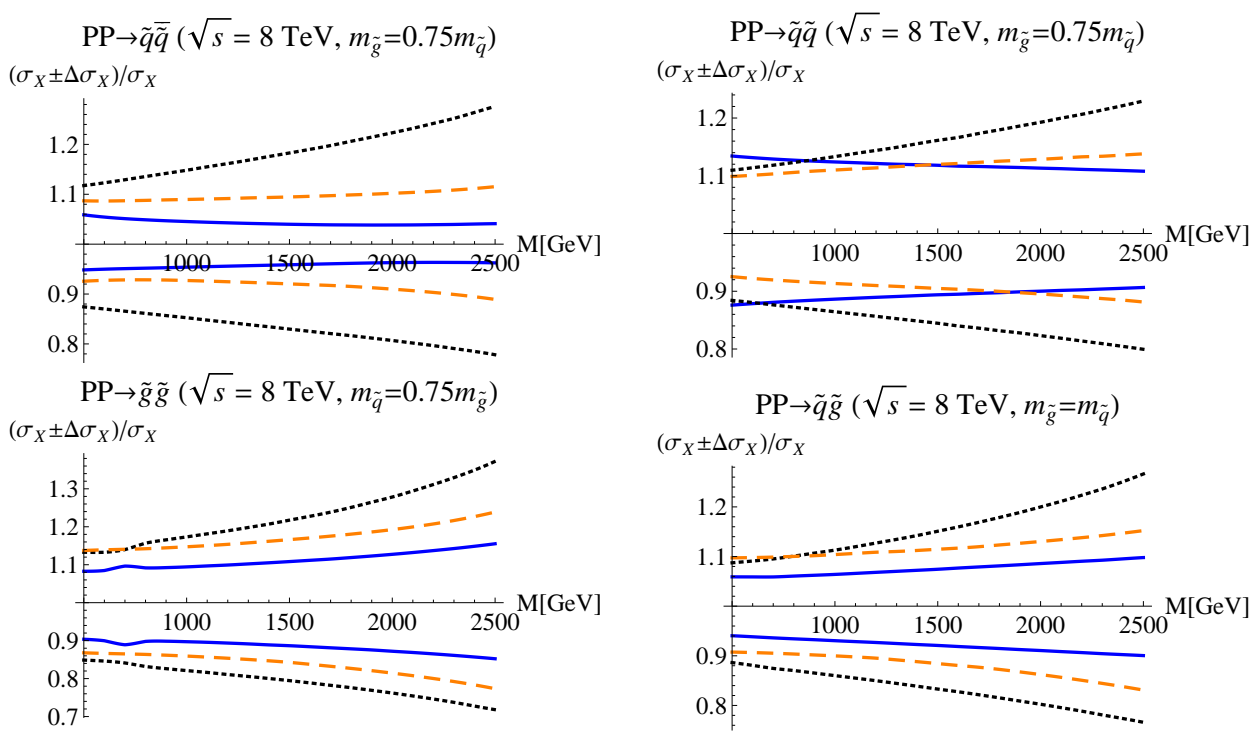

Figure 3: Total theoretical uncertainty of the NLO approximation (dotted black), NLL (dashed orange), and NNLL (solid blue) resummed results at the LHC with $\sqrt{s}=8 \mathrm{TeV}$. All cross sections are normalized to one at the central value of the scales.

the product of hard and Coulomb corrections is replaced by its expansion up to $\mathscr{O}\left(\alpha_{s}^{2}\right)$. The scale uncertainty of the NNLL predictions is estimated by varying $\mu_{f}, \mu_{h}$, and $\mu_{C}$ from half to twice their default values. We use a running soft scale $\mu_{s}=k_{s} M \max \left\{\beta^{2}, \beta_{\text {cut }}^{2}\right\}$ [24 with $k_{s}=1$. The default value of $\beta_{\text {cut }}$ is determined following [27] and the resulting uncertainty is estimated by setting $k_{s}=0.5,2$ as well as varying $\beta_{\text {cut }}$ by $\pm 20 \%$ and taking the envelope of several resummed and fixed-order approximations. As a measure of power-suppressed terms, the non-relativistic energy $M \beta^{2}$ is replaced by $E=\sqrt{\hat{s}}-2 M$. Finally, a constant term $\pm\left|h_{p p^{\prime}}^{R(1)}\right|^{2}$ is added as an estimate of unknown NNLO corrections beyond the threshold limit. The uncertainties from the various sources are added in quadrature.

Our results for the K-factors beyond NLO $K_{X}=\sigma_{X} / \sigma_{\mathrm{NLO}}$ with $X=\mathrm{NLL}$, NNLL, NNLL fixed-C, and $\mathrm{NNLO}_{\text {app }}$ for the four squark and gluino production processes are shown in Figure 2 . The results show a full NNLL correction of up to $25 \%$ relative to the NLL results. The effect of Coulomb resummation can be important in particular for squark-antisquark and gluino-pair production. The comparison to the approximate NNLO results shows that corrections beyond NNLO become sizeable beyond sparticle masses of $\sim 1.5 \mathrm{TeV}$. The $\mathrm{NNLL}_{\text {fixed-C }}$ results appear to be in good agreement with results of the Mellin-space approach to resummation [23] where a similar approximation is used. An exception is gluino-pair production, where our result differs by about $10 \%$ from the one in [23]. As can be seen in Figure 3 the relative uncertainty is reduced from up to $30 \%$ at NLO, to at most $20 \%$ at NLL and to the $10 \%$-level at NNLL.

\section{References}

[1] G. Aad et al. [ATLAS Collaboration], arXiv:1405.7875.

[2] M. Beneke, P. Falgari, J. Piclum, C. Schwinn, and C. Wever, in preparation and PoS RADCOR2013 (2014) 051 arXiv: 1312.0837. 
[3] P. Falgari, C. Schwinn, and C. Wever, $\operatorname{VHEP~1206~(2012)052~arXiv:1202.2260.~}$

[4] W. Beenakker et al., Nucl. Phys. B 492 (1997) 51 hep-ph/9610490.

[5] W. Beenakker, R. Höpker, and M. Spira, hep-ph/9611232.

[6] A. D. Martin et al., Eur. Phys. J. C 63 (2009) 189 [arXiv:0901.0002].

[7] A. Kulesza and L. Motyka, Phys. Rev. Lett. 102 (2009) 111802 arXiv:0807.2405 and Phys. Rev. D 80 (2009) 095004 [arXiv: 0905.4749 ].

[8] W. Beenakker et al., JHEP 0912 (2009) 041 [arXiv: 0909.4418 and JHEP 1008 (2010) 098 [arXiv:1006.4771].

[9] M. Beneke et al., Phys. Lett. B 690 (2010) 483 arXiv:0911.5166.

[10] W. Beenakker et al., JHEP 1201 (2012) 076 arXiv:1110.2446].

[11] M. R. Kauth et al., Nucl. Phys. B 857 (2012) 28 arXiv:1108.0361.

[12] U. Langenfeld, S.-O. Moch, and T. Pfoh, $\operatorname{HEP} 1211$ (2012) 070 arXiv: 1208.4281.

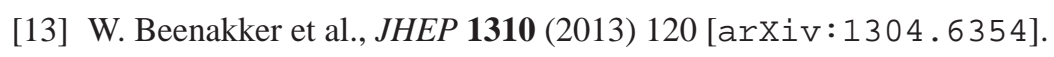

[14] G. F. Sterman, Nucl. Phys. B 281 (1987) 310:

S. Catani and L. Trentadue, Nucl. Phys. B 327 (1989) 323.

[15] N. Kidonakis and G. F. Sterman, Nucl. Phys. B 505 (1997) 321 hep-ph/9705234;

R. Bonciani et al., Nucl. Phys. B 529 (1998) 424 [hep-ph/9801375].

[16] T. Becher and M. Neubert, Phys. Rev. Lett. 97 (2006) 082001 hep-ph/0605050;;

T. Becher, M. Neubert, and G. Xu, JHEP 0807 (2008) 030 arXiv: 0710.0680.

[17] see e.g. A. H. Hoang et al., Eur. Phys. J. direct C 2 (2000) 1 [hep-ph/0001286.

[18] M. Beneke, P. Falgari, and C. Schwinn, Nucl. Phys. B 842 (2011) 414 arXiv: 10 07.5414 .

[19] K. Hagiwara and H. Yokoya, JHEP 0910 (2009) 049 arXiv: 0909.3204 ;;

M. R. Kauth, A. Kress, and J. H. Kühn, JHEP 1112 (2011) 104 arXiv: 1108.0542.

[20] U. Langenfeld and S.-O. Moch, Phys. Lett. B 675 (2009) 210 [arXiv: 0901.0802 ];

U. Langenfeld, $\operatorname{VHEP~1107~(2011)~052~[arXiv:~1011.334]];~}$

A. Broggio et al., JHEP 1307 (2013) 042 [arXiv: 1304.2411].

[21] T. Pfoh, JHEP 1305 (2013) 044 arXiv: 1302.7202].

[22] A. Broggio et al., JHEP 1403 (2014) 066 [arXiv: 1312.4540 ];

C. Kim et al., Phys. Rev. D 89 (2014) 075010 arXiv: 1401.1284 ].

[23] W. Beenakker at al., arXiv:1404.3134.

[24] M. Beneke, P. Falgari, and C. Schwinn, Nucl. Phys. B 828 (2010) 69 arXiv: 0907.1443].

[25] T. Becher and M. Neubert, Phys. Rev. D 79 (2009) 125004 [arXiv:0904.1021];

N. Kidonakis, Phys. Rev. Lett. 102 (2009) 232003 arXiv:0903.2561.

[26] M. Czakon, A. Mitov, and G. F. Sterman, Phys. Rev. D 80 (2009) 074017 arXiv: 0907.1790 .

[27] M. Beneke et al., Nucl. Phys. B 855 (2012) 695 arXiv:1109.1536].

[28] M. Beneke, A. Signer, and V. A. Smirnov, Phys. Lett. B 454 (1999) 137 hep-ph/9903260].

[29] P. Bärnreuther, M. Czakon, and P. Fiedler, JHEP 1402 (2014) 078 arXiv: 1312.6279].

[30] P. Falgari, C. Schwinn, and C. Wever, IHEP 1301 (2013) 085 arXiv:1211.3408.

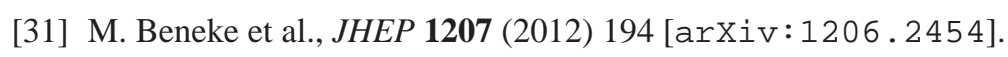

